\title{
ETHICAL ASPECTS OF HOME INFORMATICS AND TELEMATICS
}

\author{
Andy Sloane \\ Research Institute in Advanced Technology, School of Computing and IT, University of \\ Wolverhampton, U.K.
}

\begin{abstract}
Home-oriented Informatics and Telematics (HOIT) research has a number of challenges in the future. These range from the technical challenge of providing useful solutions through the ubiquitous paradigm to the cultural challenges of the $21^{\text {st }}$ century. While much research focuses on the technical processes of devices, services and systems for the home there is little reflective research that looks into the ethical components of these developments. This paper outlines some of the ethical problems that are faced by developers and researchers in this subject.
\end{abstract}

Key words: Ethics, sustainability, market forces, technology.

\section{INTRODUCTION}

The home is a concept that is rooted in many traditions and cultures of the world (Sloane, 2003). Different concepts of home are relevant in different cultures; and within these there are many different instances of "home". This has made generic study of "home-life" very difficult over the years for the many researchers that are interested in the subject. (Sloane, 2002).This variability in the manifestation of the home concept has led to many generalisations in the design and production of devices, systems and services for the home that do not give the optimum service for individuals. The rooting of the home in its culture is fundamental - it is not a standalone concept devoid of influence from the embedding social fabric, but an integral part of society, neighbourhood and culture. In many modern societies there is a move towards multiple co-existing cultures with a wide 
variety of home styles and configurations in evidence. This leads to even more disparity between the perceived usefulness of home systems design and their actual use and application. To encompass this diversity is one of the challenges of HOIT for the future, but to do so requires a more ethical approach to the development of services and systems for the home environment that will incorporate the cultural diversity in different countries, and the diversity in multi-cultural environments and foster the development of devices and system that aid and assist the user where necessary without causing more problems than they solve.

\section{MARKET-LED DEVELOPMENT}

The main source of devices, systems and services for home users is the industry that sells these to consumers. The marketing push in the industry is clearer to engage a wide range of users in the process of purchasing new equipment and systems for the home. Some technologies have been more successful than others and there are a number of historical studies that show that wider take-up is not necessarily because of superior technology (see Liebowitz and Margolis, 1995) and the outcome is often closer related to marketing, advertising and other non-technical factors rather than any technological, or even usability, superiority.

The market background document from the TEAHA project (Homega Research, 2004) gives some insight into the status of various technologies and their take-up around the world. This allows some perspective on the market for connected home products. As they point out in this report the prediction of markets is fraught with danger and simple extrapolation is not a reliable indicator of future trends in such diverse markets as home electronics and leisure equipment.

The market-led nature of this technology is also a factor that does not lead to the most ethical style of development for the systems in question. The primary goal of manufacturers is to sell goods, to make profits and to continue to operate. They do not necessarily have any "emotional capital" in the goods that they sell and promote. In fact there are a number of conflicting goals for manufacturers that do not assist the user. Amongst these are the problems of compatibility and standards.

\subsection{Compatibility issues}

There are some complex economic issues involved in the production of goods for the HOIT market, as for many other markets for electronic goods and services. Many of these issues are related to the compatibility of 
different components and systems for use in the home. The issue for companies and the goods they produce (i.e. do they produce them to be compatible with others or not?) rests on many factors and is too complex to discuss in this paper. However, the more significant side of this argument, from the home perspective, is summarized by the following (Economides, 1999):

"Society reaps significant benefits from compatibility and standardization"

It is this factor and the incompatibility of much market-driven production that are the opposing forces in the HOIT arena and that can determine the outcome of technological developments.

The compatibility of devices, systems and software is not a pre-requisite for HOIT development but it has a considerable influence on the take-up and spread of technology in the home and across cultures and countries. It is also clear that the ethical development of home systems, devices and services requires them to be developed using an ethical perspective which must account for the societal need for standardization in addition to the commercial requirement for profit. One example of how this could be done, to promote better streamed multimedia is outlined in Sloane 2002, where infrastructure developments are shown to provide better quality of broadcast.

\subsection{Standards}

The issue of standardization is quite complex. The opposing views are that: on the one hand, standards are necessary to ensure compatibility of equipment and, on the other, that standards are a restriction to the successful development of new devices and systems. Both of these viewpoints are, to some extent true. The use of standardization has helped many systems to gain a bigger share of the market and many manufacturers are able to compete in a market that is led by standardization activities. Examples of this are: the CD audio and DVD video standards. These have led to many different devices being available to users to play the standard software.

Standardisation of media standards helps both equipment manufacturers and content producers provide users with compatible devices and useable content. Indeed, without the standardization effort the market may not reach critical mass before the device or system becomes obsolete. There have been many different proprietary media standards that have faltered without the back-up of a wide variety of content to enhance sales.

The opposite problem of standards; that of restricting innovation is only problematic when the devices that are standardized become widespread and the standard becomes technically limited compared to other competing 
standards. This situation is the case with video recording equipment. There is a large-scale legacy investment in VCRs around the world and they are now much more limited than the digital replacements DVD recorders. However, there is still a need to support the VCR user with software content, consumables and maintenance for some time into the future. There are some moves to end support for VCRs by ending sales but the legacy of many old devices will continue for a number of years and there are still manufacturers supplying new devices for users.

The move from video-cassette to DVD as the medium of choice for consumers is likely to be driven by a number of factors: wider DVD content provision, compatibility with other devices such as PC drives, multi-function capabilities (e.g. on-screen viewing of photos), digital input, output quality factors, price (although VCRs are currently much cheaper than DVD recorders), and media cost (DVD+RW and Video-cassette prices are roughly comparable). However, one of the most useful and attractive characteristics of using discs is the indexing and random-access capabilities of the devices. All these factors and the market push of the manufacturers is likely to see the replacement of VCRs by DVD devices in a fairly short time.

The next problem that will be faced by home IT users is then likely to be the recording of family archives of legacy content onto new media. This has been an issue in the past, albeit on a smaller scale, when the introduction of home video recorders made home use of cine film unattractive. Some content was moved onto video-cassette by specialist companies. Upgrading to digital formats will see more legacy content being moved to new media systems.

\section{ETHICAL DESIGN}

There have been a number of studies that have used co-operative design methods (for example, Kristensen et al, 2003) these are perhaps the most appropriate for the design of home devices as they involve the end-user in the design process, although there are problems with the application of any methods to the design of home devices.

However, there remains the problem of how the design of home systems can be made more ethical. The initial problem of how to design a home artefact needs to be replaced by an extra stage of analysis that asks the fundamental question "Should this device or system be designed and made?" and if it is, "How should it be made so that it can be used ethically?" and finally "Is it possible to make an ethical version by excluding or enhancing certain functionality?" 
With the use of a comprehensive ethical analysis of the situation of the user and the device, put into the context of the home, devices can be designed to include a more ethical dimension for the type of device that is in use by people for a significant amount of their free time. The idea of an ethical dimension to home-system design will be explored in the following sections where the design of home devices, home services and the status of the user will be outlined and discussed

\subsection{Home devices}

Many home devices are developed to enhance the users' leisure time activities; others are developed to be labour-saving devices, some devices incorporate an element of both functions. There are a number of design considerations that are needed when developing devices for the home. These will depend on the function and use of the device under consideration. In addition, for an ethical perspective to be included in the design process an extra stage will need to be added that takes a wider perspective at different stage of the development process. Incorporating ethical aspects into the design process is not easy and many designs assume that the devices designed will be used in particular ways (Robertson, 2004). In the home context there are other factors that need consideration. Freedom to experiment and use devices in ways that are not pre-determined by design are aspects of device development that are useful to leisure users. There are also aspects of design that are ethically questionable when wider societal issues are included in the design process. For example, the production of violent computer games is a problem area where access to such software is not restricted, reportedly causing further aggression if the users frequently play the games (Bushman 2002).

There are also areas of device design that may cause ethical dilemmas in that perceived use is not necessarily in the interests of the consumer. A useful case of this to analyse is the ubiquitous use of the remote control to control devices $n$ the home. There are many situations when a remote device is useful, and it does allow easy and simple control of television and other household devices. However, for some of the population (e.g. the morbidly obese), excessive use of the remote control compounds their inactivity and can exacerbate their health problem. Indeed, it has been stated that the general population would benefit from the removal of remote control devices to increase activity levels. Is this a case for ethical design? 


\subsection{Services}

The provision of digital services to the home is also an area where ethics can play a part in enhancing the experience for users and allowing a better interaction with the service provider. There are now a multitude of different services aimed at the home user with many different types of interaction allowed with the various services. Much if what is sold to home dwellers is done so under the banner of leisure and entertainment. However, this is essentially a service that only caters for a small minority of viewers in that they fit the unique market research categories used to determine programme output by the various providers. This usually entails a diet of popular work that does little to enhance the television experience and does not venture into new territory or allow for much cultural diversity. As most of the revenues of the television and other service providers depends on high volume this is not surprising, but what is now possible is the expansion of audio-visual programming to include Internet delivery in some cases which would allow the more esoteric and culturally diverse offering for home users.

\subsection{Who is the user?}

It may seem to be a strange question to ask but who is the user in this context. The home is a place where people live-it therefore encompasses all users to a certain extent. This is a basic problem for home-based research i.e. what is a definition of "the user"?

Homes consist of many different, culturally-dependent configurations. Any one home is different from any other sometimes in quite profound ways - in its structure, operation, context, organisation and use. The ethical approach would be to incorporate this diversity in the design process. This is obviously a very difficult process, as user-groups in the home encompass all age groups, many different states of health and well-being and different needs, wants and desires-all within the same home.

\subsection{The Internet}

The Internet is now the medium of choice for the delivery of online services to the home. The Internet has developed from a computer network for research and the military, into a global network incorporating both commercial and non-commercial interest and a near-complete range of electronic human activity. This has led to some disquiet in certain areas. Many groups of Internet users do not want to interact with some of the material that is contained on the World Wide Web. Examples are: the case of 
separating pornography and young Internet users, and the promotion of violence or illegal drug use.

There are many areas of human life that are not suitable for wider promulgation, but legal restrictions have little effect on an electronic environment that spans continents and where they have little long-term effect. The Internet has allowed users to interact on a much wider scale, to enhance their leisure activity with a wider range of sources of knowledge and to pursue new interests that were not possible before the advent of the worldwide Internet. This has led to a situation where the Internet is now indispensable for many home-based users as it allows them to interact both socially and for commercial activity in a way that is becoming symptomatic of the $21^{\text {st }}$ century.

The growth of the Internet is also one reason that it has become difficult to govern. Multi-national co-operation is required to enforce any effective features on the network and this is unlikely to be a feature of any future embodiment of the Internet or the WWW. Ethical development of Internet services may be left out of plans for governance but it does not need to be excluded from user-level service provision. If user demand is sufficient for value-added services to be economically provided they will become more widely available.

\subsection{Future devices and services}

For future devices the design process can be adapted to incorporate some of the ideas outlined so far: greater inclusivity, wider applicability, more ethical input to functions and design and more diverse approaches to service provision. These changes will not be easy or quick to implement; a radical overhaul of design practice and service provision needs to be undertaken and there may be resistance from manufacturers and consumers to some developments if the changes are not included with a collateral change in the legal framework, the social expectations of the home dweller and the societal organisation of commercial entities.

\section{CONCLUSIONS}

Many developments have changed the face of the home environment over the years since information and communication technology became a consumer item. The growth of information services and devices has allowed home-users to extend their leisure and the facilities they use into a virtual world. This new paradigm for home users has caused problems for many of them. These problems are partly caused by the lack of a sufficiently ethical 
dimension into the design of devices and services for home users. A more ethical approach would see more inclusivity, more cultural diversity and better quality of devices and services for home-based users.

The ethical approach is, however, not necessarily a simple and easy route to follow. Human nature militates against some of the more appropriate ethical developments and cross-cultural differences are also hard to combine into an approach that is inclusive. There are, therefore many barriers to overcome on the way to an ethical approach to home systems design, but there are a number of areas that can be used as a starting point. It may be too late to influence the design of existing systems but future systems can be influenced by a more ethical approach - it is still to be seen whether it will happen.

\section{REFERENCES}

Bushman, B. J., \& Anderson, C. A. (2002). "Violent video games and hostile expectations: A test of the General Aggression Model". Personality and Social Psychology Bulletin, 28, 1679-1689.

Economides N (1999) "Competition and Vertical Integration in the Computing Industry", in Eisenach J. A. and. Lenard T M (eds.), Competition, Innovation, and the Role of Antitrust in the Digital Marketplace, Kluwer Academic Publishers 1999

Homega Research (2004) "The worldwide markets for the Connected home: status and trends", TEAHA background document, Available from http://www.teaha.org/project_outputs.php (Downloaded $9^{\text {th }}$ November 2004)

Kristensen J F, Eriksen M A, Iversen O S, Kanstrup A M, Nielsen C and Petersen M G (2003) "Young People in Old Cars - Challenges for Cooperative Design", Electronic Proceedings of the 26th Information Systems Research Seminar in Scandinavia/IRIS26, Haikko Manor, Finland, August 9-12, 2003.

Liebowitz S J and Margolis S.E (1995), "Path Dependence, Lock-In and History "Journal of Law, Economics and Organization.

Robertson T (2004) "Ethical issues in interaction design" http://research.it.uts.edu.au /idwop/downloads/Robertson/Ethics\&IT.pdf. Viewed and downloaded 10/12/2004.

Sloane A (2000) "Infrastructure issues for Internet broadcasting to home-based users", in Beardon, Munari and Rasmussen (Eds.), "Computers and Networks in the Age of Globalisation", Kluwer, Boston, ISBN 0-7923-7253-0, pp187-196.

Sloane A (2002) "Methodologies for studying the home user" - World Computer Congress, Montreal, August 2002.

Sloane A (2003) "The Internet in the home: Changing the domestic landscape" in Stephanides, C. (ed.). Proceedings of HCII2003, Crete, June 22-27. 